\title{
New approaches for the design of DNA vaccine vectors delivered by lactic acid bacteria
}

\author{
Keywords: lactic acid bacteria, DNA vaccine, vector design
}

\section{Introduction}

Lactic Acid Bacteria (LAB) have been bioengineered for mucosal delivery of prophylactic, pro-inflammatory and useful molecules that include bioactive peptides, cytokines, enzymes, allergens and DNA. One of the many benefits that have been attributed to these has been the modulation of immune responses. Regarding LAB as a means of delivery, it is important to highlight that this bacteria have the ability to resist gastric and bile juices allowing their survival and transit through the gastrointestinal $\operatorname{tract}^{1,2}$ and conferring them the capability to delivery molecules directly at the intestinal mucosal surface. The Food and Drug Administration (FDA) of the U.S.A. consider Lactobacilli and Lactococcus lactis safe or "GRAS" (Generally Recognized as Safe), and according to with European Food Safety Authority (EFSA), these species have fulfilled the criteria of the competent Qualified Presumption of Safety (QPS) status. Increase knowledge in genetic engineering technologies have made it possible to incorporating new genes, as well as modifying the metabolic functions of these important microorganisms which has lead to improvements in health and food technology., The oral administration of live recombinant microorganisms such as LAB can be considered as bio-drugs or biopharmaceuticals. This strategy can be used not only in the prevention or in the treatment of different diseases, but also in the development of innovative drugs and vaccines. ${ }^{5,6}$ DNA vaccines are constructions based on plasmids that combine sequences that allows its replication in different bacterial species (including E. Coli and LAB) with others that are necessary to express the transgenic of interest in vertebrate cells after delivery. ${ }^{7}$ These kind of vaccines using LAB as delivery vehicles are extremely safe and because they cannot revert to a disease causing form (as occurs with viral vector) since they only encode and express the target antigens and not virulence factors. ${ }^{8}$ Only a handful of groups are performing DNA vaccine design vectors that can be delivered by LAB. One is being led by Dr. Desai; who recently published the construction of the $p$ PERDBY plasmid, in which the reporter gene is in the backbone of the plasmid allowing the cloning of the gene of interest in frame with a reporter gene. ${ }^{9}$ Our group, led by Dr. Azevedo developed in 2009, the $p$ Valac vector, the first vector designed for DNA vaccines to be delivered by LAB.$^{10}$ Recently we have published a new DNA vaccine vector called pExu. ${ }^{11}$ The $\mathrm{pExu}$ vector has some attractive characteristics, such as containing the theta origin replication which offers a higher structural and segregation stability, and has showed exceptional results in In vivo test, ${ }^{11}$ and can also stably maintain large heterogonous DNA inserts. ${ }^{12,13}$ All of these plasmids are only being used in "proof- of- concept" studies since these vectors having antibiotic resistance markers that for legal and ethical reasons are not acceptable which might compromise their applications in health treatments. For genetically modified $\mathrm{LAB}$ to be used, the development of food-grade cloning systems and biological containment systems are part of the solution for these to be approved for use in human trials. Johansen (1999) defined foodgrade recombinant microorganism as those that contain DNA from the same genus or from other GRAS microorganisms. ${ }^{14}$ Some food-grade

\author{
Volume 2 Issue 5 - 2017
}

\author{
Pamela Mancha-Agresti,' Jean Guy \\ Leblanc, ${ }^{2}$ Vasco Azevedo,' Mariana Martins \\ Drumond ${ }^{1,3}$ \\ 'Instituto de Ciências Biológicas, Universidade Federal de Minas \\ Gerais, Brazil \\ ${ }^{2}$ CERELA-CONICET, Centro de Referencia para Lactobacilos, \\ Argentina \\ ${ }^{3}$ CEFET - Centro Federal de Educação Tecnológica de Minas \\ Gerais, Brazil
}

Correspondence: Vasco Azevedo, UFMG, Federal University of Minas Gerais, Belo Horizonte, Minas Gerais, Brazil, Email vasco@icb.ufmg.br

Received: June 13, 2017 | Published: December 05, 2017

vectors have been constructed for $\mathrm{LAB}$, but only for the production of heterologous proteins, ${ }^{15-17}$ not to deliver foreign DNA. The design of these vectors is based on the replacement of antibiotics resistance selection markers; however, other properties need to be considered in order to construct effective food-grade vectors to be used as DNA vaccines. The size, the number of copies of the plasmid, the ability to correctly translate the protein of interest in host cell, as well as their low capacity of recombination with host's DNA are some characteristics that need to be considered. The use DNA delivery vectors by $\mathrm{LAB}$ does not require complex technologies and can even replace the industrial production of certain proteins that require expensive purification protocols since the host cells would produce the protein of interest for themselves.

\section{Discussion}

The necessity to go beyond the "proof-of concept" stage needs to be resolved. Plasmid replication has an important impact on DNA vaccines. Vectors with RCR replicas, due to accumulation of ss-DNA intermediates, usually have low segregational stability ${ }^{18,19}$ as they appear to lack a partitioning function. Plasmids are probably randomly distributed over daughter cells, ${ }^{20}$ and the insertion of external DNA may contribute to reducing their stability even more. ${ }^{21}$ On the other hand, theta-replicating plasmids don't produce ss-DNA intermediates, offering better structural and segregational stability and allowing to maintain bigger foreign DNA inserts ${ }^{12,13}$ facilitating cloning techniques. The copy number of the plasmid is of great importance for plasmid delivery strategies and it is obvious that higher number lead to better success rates. ${ }^{22}$ Plasmids with theta origin replication have higher copy numbers, are more structurally and segregationally stable and thus constitute an interesting tool for improve plasmid delivery.

The size of the vector and the plasmid copy number of bacteria are closely related to the replication origin, where the biggest size has been described for theta replication. Although plasmid size is relevant on gene transfer, it is assumed that smaller plasmids are can more easily find the nucleus through the viscous cytosolic environmental, 
and easily achieve the cell nucleus, as well as for manipulation like cloning strategies and transformations steps in lactococci. ${ }^{10,23,24}$ Furthermore, the stability of the vector is very significant at the time of choosing DNA vaccines. We were able to show this with an in vivo test using the pExu vector $(\mathrm{Kb} \mathrm{8,000)}$. Although it is a large plasmid, it shows excellent expression of eGFP in eukaryotic cells. ${ }^{11}$ Most researchers encourage the minimization of vectors size, by removing extra non-functional sequences like those that encode ORFs that may be expressed in the host organism (cryptic ORFs, for example). ${ }^{25,26}$ Other authors showed that small vectors can lead to higher transgene expression, making the transfection into the host cells more effective. ${ }^{27,28}$

The substitution of the antibiotic selection marker can also increase the expression in host organism. ${ }^{29}$ In fact, this issue is one of the most relevant and the current aim for the design food-grade vectors to be used in human. Selection markers are of great importance in recombinant DNA technology. The FDA and the European Union (EU) have supplied guide documents with information and considerations about vector design for plasmid vectors planned for human use. ${ }^{30-32}$ Antibiotics resistance genes as a selection marker are not acceptable for clinical trials due to the risk that the vector strains could spread into the environment, as well as the potential horizontal gene transfers possibility which could provide pathogenic bacteria with resistance to antibiotics that are used for patient treatment. ${ }^{33}$ In fact, the design of antibiotic-free plasmid, either gene or protein delivery using bacterial would be advantageous because it would increase their biosafety. With this aim, several alternatives are being considered, such as immunity markers and bacteriocin production; ${ }^{31,32}$ however, studies showed these had limited efficacy. ${ }^{26,34}$ One promising strategy is auxotrophy: modifying the bacterial strains by generating a nonsense point mutation or deletion of an essential chromosomal gene. To restore the growth of the bacteria, a plasmid carrying the deleted/ mutated gene would be introduced together with the therapeutic gene to be expressed. Several genes have been studied for this approach by LAB ${ }^{35,36}$ The challenge is now developing auxotrophic strains to be used as DNA vaccines that can be efficiently delivered by LAB.

\section{Conclusion}

LABS have been used for a long time in food to enhance flavors as well as to offer health benefits and has nowadays seen a rise interest in their use in medicine. Nevertheless, it is necessary to improve new generation vector designs that could be used in novel biotechnology products such as DNA vaccine. Optimizing vector construction with increased transgene expression, the removal of antibiotic resistance markers, maintaining plasmid stability and safety for host are targets that need to be reached. It is also important to determine the best delivery platforms to ensure the safety of DNA vaccines for prophylactic or therapeutic uses in human diseases.

\section{Acknowledgements}

None.

\section{Conflict of interest}

Author declares that there is no conflict of interest.

\section{References}

1. Wells JM, Mercenier A. Mucosal delivery of therapeutic and prophylactic molecules using lactic acid bacteria. Nat Rev Microbiol. 2008;6(5):349-362.
2. Wells JM. Immunomodulatory mechanisms of lactobacilli. Microb Cell Fact. 2011;10(1):S17.

3. Bermúdez Humarán LG, Aubry C, Motta JP, et al. Engineering lactococci and lactobacilli for human health. Curr Opin in Microbiology. 2013;16(3):278-283

4. LeBlanc JG, Aubry C, Cortes Perez NG, et al. Mucosal targeting of therapeutic molecules using genetically modified lactic acid bacteria: an update. FEMS Microbiol Lett. 2013;344(1):1-9.

5. D Silva I. Recombinant Technology and Probiotics. International Journal of Engineering and Technology. 2011;3(4):288-293.

6. Blanquet S, Marol Bonnin S, Beyssac E, et al. The "biodrug" concept: an innovative approach to therapy. Trends Biotechnol. 2001;19(10):393-400.

7. Williams J. Vector Design for Improved DNA Vaccine Efficacy, Safety and Production. Vaccines (Basel). 2013;1(3):225-249

8. Premenko Lanier M, Rota PA, Rhodes GH, et al. Protection against challenge with measles virus (MV) in infant macaques by an MV DNA vaccine administered in the presence of neutralizing antibody. Journal of Infectious Diseases. 2004;189(111):2064-71.

9. Yagnik B, Padh H, Desai P. Construction of a new shuttle vector for DNA delivery into mammalian cells using non-invasive Lactococcus lactis. Microbes Infect. 2016;18(4):237-244.

10. Guimarães V, Innocentin S, Chatel JM, et al. A new plasmid vector for DNA delivery using lactococci. Genet Vaccines Ther. 2009;7:4.

11. Mancha Agresti P, Drumond MM, Rosado Carmo FL, et al. A New Broad Range plasmid for DNA Delivery in Eukaryotic Cells Using Lactic Acid Bacteria: In vitro and In vivo assays. Mol Ther Methods Clin Dev. 2016;4:83-91.

12. Kiewiet R, Bron S, De Jonge K, et al. Theta replication of the lactococcal plasmid pWVO2. Mol Microbiol. 1993;10(2):319-327.

13. De Vos WM, Simons GFM. Gene cloning and expression systems in Lactococci. Genetics and Biotechnology of Lactic Acid Bacteria Dordrecht. 1994:52-105.

14. Johansen E. Genetic engineering. Modification of bacteria. London: Academic Press; 1999.

15. Henrich B, Klein JR, Weber B, et al. Food-Grade Delivery System for Controlled Gene Expression in Lactococcus lactis. Applied Environmental Microbiology. 2002;68(11):5429-5436.

16. Leenhouts K, Bolhuis A, Venema G, et al. Construction of a food-grade multiple-copy integration system for Lactococcus lactis. Appl Microbiol Biotechnol. 1998;49(4):417-423.

17. Simões-Barbosa A, Abreu H, Silva Neto A, et al. A food-grade delivery system for Lactococcus lactis and evaluation of inducible gene expression. Appl Microbiol Biotechnol. 2004;65:61-67.

18. Posno M, Leer RJ, van Luijk N, et al. Incompatibility of Lactobacillus Vectors with Replicons Derived from Small Cryptic Lactobacillus Plasmids and Segregational Instability of the Introduced Vectors. Applied and Environmental Microbiology. 1991;57(6):1822-1828.

19. Vujcic M, Topisirovic L. Molecular analysis of the rolling-circle replicating plasmid pA1 of Lactobacillus plantarum A112. Appl Environ Microbiol. 1993;59(1):274-280.

20. Novick R. Plasmid incompatibility. Microbiol Rev. 1987;51(4):381-395.

21. Gruss A, Ehrlich SD. The family of highly interrelated single-stranded deoxyribonucleic acid plasmids. Microbiol Rev. 1989;53(2):231-241.

22. Mariat D, Robert V, Langella P, et al. Plasmid transfer efficiency using Lactoccocus lactis strains depends on invasiveness status but also on plasmid copy number. FEMS Microbiol Lett. 2017;364(14) 
23. Escriou V, Ciolina C, Helbling Leclerc A, et al. Cationic lipid-mediated gene transfer: analysis of cellular uptake and nuclear import of plasmid DNA. Cell Biology and Toxicology. 1998;14(2):95-104.

24. Escriou V, Carrire M, Bussone F, et al. Critical assessment of the nuclear import of plasmid during cationic lipid-mediated gene transfer. $J$ Gene Med. 2001;3(2):179-187.

25. Li C, Goudy K, Hirsch M, et al. Cellular immune response to cryptic epitopes during therapeutic gene transfer. Proc Natl Acad Sci US A. 2009;106(26):10770-10774.

26. Schirmbeck R, Riedl P, Fissolo N, et al. Translation from cryptic reading frames of DNA vaccines generates an extended repertoire of immunogenic, MHC class I-restricted epitopes. J Immunol. 2005; 174(8):4647-4656.

27. Kreiss P, Cameron B, Rangara R, et al. Plasmid DNA size does not affect the physicochemical properties of lipoplexes but modulates gene transfer efficiency. Nucleic Acids Res. 1999;27(19):3792-3798.

28. Yin W, Xiang P, Li Q. Investigations of the effect of DNA size in transient transfection assay using dual luciferase system. Anal Biochem. 2005;346(2):289-294.

29. Oliveira PH, Mairhofer J. Marker-free plasmids for biotechnological applications? implications and perspectives. Trends Biotechnol. 2013;31(9):539-547.
30. Rockville. Guidance for Industry: Considerations for Plasmid DNA Vaccines for Infectious Disease Indications. Food and Drug Administration. USA; 2007.

31. EMA. Note for Guidance on the Quality, Preclinical and Clinical Aspects of Gene Transfer Medicinal Products. EU European Medicines Agency; 2001.

32. EMU. Presence of the Antibiotic Resistance Marker Gene nptII in GM Plants and Food and Feed Uses. EU European Medicines Agency; 2007.

33. Vandermeulen G, Marie C, Scherman D, et al. New generation of plasmid backbones devoid of antibiotic resistance marker for gene therapy trials. Mol Ther. 2011;19(11):1942-1949.

34. Luke JM, Vincent JM, Du SX, et al. Improved antibiotic-free plasmid vector design by incorporation of transient expression enhancers. Gene Ther. 2011;18(4):334-343.

35. Steidler L. Genetically engineered probiotics. Best Pract Res Clin Gastroenterol. 2003;17(5):861-876.

36. Landete JM. A review of food-grade vectors in lactic acid bacteria: from the laboratory to their application. Crit Rev Biotechnol. 2017;37(3):296-308. 